\title{
Genetic Diversity of Porcine Reproductive and Respiratory Syndrome Virus and Evaluation of Three One-Step Real-Time RT-PCR Assays in Korea
}

Go-Eun Shin

Animal and Plant Quarantine Agency

Ji-Young Park

Animal and Plant Quarantine Agency

Kyoung-Ki Lee

Animal and Plant Quarantine Agency

Mi-Kyeong Ko

Animal and Plant Quarantine Agency

Bok-Kyung Ku

Animal and Plant Quarantine Agency

Choi-Kyu Park

Kyungbuk National University

Hye-Young Jeoung ( $\nabla$ jhy98@korea.kr)

Animal and Plant Quarantine Agency

\section{Research Article}

Keywords: porcine reproductive and respiratory syndrome virus (PRRSV), genetic diversity, evaluation, diagnostic method

Posted Date: December 29th, 2021

DOI: https://doi.org/10.21203/rs.3.rs-1149203/v1

License: (1) (i) This work is licensed under a Creative Commons Attribution 4.0 International License. Read Full License 


\section{Abstract \\ Background}

Porcine reproductive and respiratory syndrome virus (PRRSV) has caused huge economic losses in the global swine industry. Frequent genetic variations in this virus cause difficulties in controlling and accurately diagnosing PRRSV.

\section{Methods}

In this study, we investigated the genetic characteristics of PRRSV-1 and PRRSV-2 circulating in Korea from January 2018 to September 2021 and evaluated three one-step real-time reverse transcription polymerase chain reaction (RT-PCR) assays.

\section{Results}

A total of 129 lung samples were collected, consisting of 47 samples for PRRSV-1, 62 samples for PRRSV-2, and 20 PRRSV-negative samples. Nucleotide sequence analysis of open reading frames (ORFs) 5, ORF6, and ORF7 genes from PRRSV samples showed that PRRSV-1 belonged to subgroup A (43/47, $91.49 \%)$ and subgroup $C(4 / 47,8.51 \%)$, whereas PRRSV-2 was classified as lineage $1(25 / 62,40.32 \%)$, Korean lineage (Kor) C (13/62, 20.97\%), Kor B (10/62, 16.13\%), lineage $5(9 / 62,14.52 \%)$, and Kor A (5/62, $8.06 \%)$. Amino acid sequence analysis showed that the neutralizing epitope and T cell epitope of PRRSV1 , and the decoy epitope region and hypervariable regions of PRRSV-2 had evolved under positive selection pressure. In particular, the key amino acid substitutions were found at positions 102 and 104 of glycoprotein 5 (GP5) in some PRRSV-2, and at positions 10 and 70 of membrane protein (M) in most PRRSV-2. In addition, one-step real-time RT-PCR assays, comprising two commercial tests and one test recommended by the World Organization for Animal Health (OIE), were evaluated.

\section{Conclusion}

The results revealed that two of the real-time RT-PCR assays had high sensitivities and specificities, whereas the real-time RT-PCR assay of the OIE had low sensitivity due to mismatches between nucleotides of Korean PRRSVs and forward primers. In this study, we genetically characterized recent PRRSV occurrences and evaluated three one-step real-time RT-PCR assays used in Korea.

\section{Background}

Porcine reproductive and respiratory syndrome virus (PRRSV) is an enveloped, single-stranded positivesense RNA virus belonging to the family Arteriviridae of the order Nidovirales. PRRSV causes reproductive failure in sows and respiratory distress in pigs of all ages, resulting in significant economic losses for the 
swine industry worldwide $(1,2)$. The PRRSV genome contains 10 open reading frames (ORFs), including ORF1a, 1b, 2a, 2b, 3, 4, 5a, 5, 6, and $7(3,4)$. ORF1a and ORF1b code for two large polyproteins that are generate 14 nonstructural proteins (4). Eight structural genes (ORF2a, ORF2b, ORF3-7, and ORF5a) encode structural proteins, including glycoprotein (GP) 2, small envelope (E), GP3, GP4, GP5, membrane $(M)$, nucleocapsid $(N)$, and ORF5a proteins, respectively $(5,6)$. PRRSV can be divided into two genotypes: European PRRSV type 1 (PRRSV-1) and North American PRRSV type 2 (PRRSV-2). The two prototype genomes, the Lelystad strain for PRRSV-1 and VR-2332 strain for PRRSV-2, share approximately $60 \%$ homology in their nucleotide sequences (7). In Korea, PRRSV-2 has spread rapidly since its first report in 1994 (8), and PRRSV-1 was identified in 2005 (9). Genetic diversity and phylogeny have been reported based on genetic analysis of ORF5 sequences of PRRSVs prevailing in Korea (10-13). The Korean PRRSV1 isolates belong only to subtype 1, whereas Korean PRRSV-2 isolates are classified as lineages 1, 4, 5, and Korean lineages (Kor) A, B, and C $(11,13,14)$.

The ORF5 sequence of PRRSV has been widely used to study phylogeny, genetic variation, and molecular epidemiology (15). Many PRRSVs that were genetically and geographically differentiated, were classified into subtypes 1-4 from PRRSV-1 and lineages 1-9 from PRRSV-2 $(3,16)$. ORF6 encodes the most conserved structural protein of PRRSV. The phylogenetic tree derived from ORF7 resembles the tree derived from the full-length genomes of PRRSV (17). The conserved regions of ORF6 and ORF7 are often used as target regions for PRRSV detection by nucleotide-based assays (18-22).

ORF5 encodes a highly variable envelope protein, GP5, which plays an important role in viral infectivity and contains immunological domains related to viral neutralization $(23,24)$. GP5 and M protein, two major envelope proteins, form a disulfide-linked heterodimer or a disulfide-linked multimer that is essential for virion formation $(25,26)$. GP4, GP5, and M proteins induce neutralizing murine monoclonal antibodies (MAbs). In particular, MAbs recognizing GP5 neutralize PRRSV more effectively than other MAbs (27). Therefore, GP5 has been considered a major target protein for vaccine design as it is involved in the production of neutralizing antibodies, followed by protection against PRRSV (28). The nonneutralizing epitope of PRRSV is highly immunodominant and exhibits some features of decoy epitopes, which have been demonstrated to inhibit recognition of neutralizing epitopes in several viral infections (28).

Nucleic acid-based diagnostic methods have been commonly used to diagnose PRRSV owing to their sensitivity, specificity, and relatively rapid test times (29-31). However, RNA viruses, such as PRRSV and swine influenza virus (SIV), have high mutation rates, rapid evolution, and genetic variability, these complicate the development of reliable diagnostic methods (32-35). Many studies have shown that genetic differences or mismatches between nucleotides of PRRSV and the primers in molecular-based assays can lead to false results (36-39). Therefore, the continuously increasing genetic diversity of PRRSV with the emergence of new strains dictates the need for an accurate diagnosis.

In this study, we investigated the genetic diversity of PRRSVs circulating in Korea through phylogenetic analysis and amino acid analysis from January 2018 to September 2021 and evaluated three one-step 
real-time reverse transcription polymerase chain reaction (RT-PCR) assays used in Korea.

\section{Methods}

\section{Clinical sample and detection of PRRSV}

A total of 129 lung samples submitted to the Diagnostic Division of the Animal and Plant Quarantine Agency (APQA) to diagnose swine disease, were collected from January 2018 to September 2021. The samples were collected from farms across all Korean provinces and included mainly clinical signs of PRRS such as acute respiratory disease in growing pigs or late-term abortion in sows. All lung tissue samples were homogenized with alpha modification of Eagle's minimum essential medium (EMEM) (Gibco, Grand Island, NY, USA) containing 1\% antibiotic (Gibco). Viral RNA was extracted from the supernatant of tissue homogenates using the RNeasy Mini Kit (QIAGEN, Hilden, Germany) according to the manufacturer's instructions. Commercial VDX ${ }^{\circledR}$ PRRSV HP MP RT-PCR and NA/EU Typing Nested PCR (Median Diagnostics, Gangwon, South Korea) were used for detecting and genotyping PRRSV.

\section{Sequencing and phylogenetic analysis of ORF5, ORF6, and ORF7 of PRRSV}

ORF5, ORF6, and ORF7 were amplified from PRRSV-positive samples by RT-PCR with specific primer sets (Table 1). RT-PCR amplification was performed under the following conditions: reverse transcription for $30 \mathrm{~min}$ at $50^{\circ} \mathrm{C}$, and termination of reverse transcription for $15 \mathrm{~min}$ at $95^{\circ} \mathrm{C}$, followed by 35 cycles of denaturation, annealing, and extension for $30 \mathrm{~s}$ at $94^{\circ} \mathrm{C}, 30 \mathrm{~s}$ at $55^{\circ} \mathrm{C}, 50 \mathrm{~s}$ at $72{ }^{\circ} \mathrm{C}$, respectively, and a final extension of $10 \mathrm{~min}$ at $72^{\circ} \mathrm{C}$. The PCR products were sequenced by commercial sequencing service company (Macrogen, Daejeon, South Korea). Reference strains, such as global PRRSV strains, Korean PRRSV strains, and two PRRSV prototype strains (Lelystad and VR2332) obtained from National Center for Biotechnology Information (NCBI), were included in the dataset for phylogenetic analysis. A phylogenetic tree was generated by maximum likelihood analysis using the Kimura two-parameter model (K2P) with MEGA 7.0 (Pennsylvania State University, State College, Pennsylvania, USA), and was evaluated by 1,000 bootstrap replicates. The Markov Chain Monte Carlo (MCMC) algorithms implemented in the BEAST v1.7.5 package was used to estimate the substitution rate per site per years (s/s/y) of the Korean PRRSV strain from 1997 to 2021. The dataset consisted of a total of 238 PRRSV-1 ORF5 sequences and 319 PRRSV-2 ORF5 sequences including Korean PRRSV reference strains available in $\mathrm{NCBI}$ and ORF5 sequences obtained in this study. Evolutionary rate was estimated using the relaxed molecular clock model with GTR $+\Gamma 4$ mixed substitution according to a previous study (40).

\section{Amino acid analysis of GP5 and M protein}

Amino acid analysis between lineages and sequence entropy at each codon indicating amino acid diversity was conducted according to a previous study (41). Briefly, graphical sequence logos for each lineage were generated using the WebLogo tool (http://weblogo.berkeley.edu/) and sequence entropy was generated using the Shannon Entropy-One tool implemented in the HIV database tool (https://www.hiv.lanl.gov/). To determine the action of selection pressure on the structural proteins of 
PRRSV-1 and PRRSV-2, site-by-site selection at single codon sites of each structural protein was estimated using the mixed-effects maximum likelihood model of evolution available at DataMonkey (http://www.datamonkey.org/) $(42,43)$. Sites with a $p$-value $\leq 0.05$ were inferred to be positively selected.

\section{One-step real-time RT-PCR assays}

Two commercially available certified one-step real-time RT-PCR assays ( $A$ and B tests) are the most commonly used for detecting PRRSV in Korea. Test $A$ and $B$ were performed using each kit provided under the same lot number. The one-step real-time RT-PCR ( $C$ test) is recommended in the OIE manual of diagnostic tests (44) and is used by a private animal disease diagnostic center. The $C$ test was performed using the QuantiNova Probe RT-PCR kit (QIAGEN). The reaction mix was prepared using $10 \mu \mathrm{L}$ of 2x probe RT-PCR Master Mix, $0.2 \mu \mathrm{L}$ of QN Probe RT-Mix, $3 \mu \mathrm{L}$ of the final concentration of forward/reverse primer and probe mix according to a previously described protocol (45), $2 \mu \mathrm{L}$ of RNase-free water, and $5 \mu \mathrm{L}$ of RNA. Reactions were performed according to the manufacturer's instructions.

\section{Sensitivities and specificities of each assay using reference strains}

The sensitivities of the assays were evaluated using two reference strains, the Lelystad strain for PRRSV1 and LMY strain (GenBank No. DQ473474) for PRRSV-2. Lelystad virus $\left(10^{5.0} 50 \%\right.$ tissue culture infective dose per $\left.\mathrm{ml}\left[\mathrm{TCID}_{50} / \mathrm{ml}\right]\right)$ and LMY virus $\left(10^{5.2} \mathrm{TCID}_{50} / \mathrm{ml}\right)$ were serially 10 -fold diluted from $10^{5}$ to $10^{-1} \mathrm{TCID}_{50} / \mathrm{ml}$ and applied to each one-step real-time RT-PCR. To estimate diagnostic specificity, other respiratory viruses, such as porcine circovirus type 2 and type 3 (PCV2 and PCV3), classical swine fever virus (CSFV), porcine parvovirus (PPV), and SIV were tested for cross-reactivity.

\section{Clinical evaluation of three one-step real-time RT-PCR assays}

The three one-step real-time RT-PCR assays were evaluated for 129 clinical samples, and PRRSV-positive samples were determined by sequencing. In order to eliminate PCR inhibition of total RNA extracted from tissue samples, false-negative samples were diluted 10 -fold in five concentrations $\left(10^{-1}, 10^{-2}, 10^{-3}, 10^{-4}\right.$, and $\left.10^{-5}\right)(46,47)$. The threshold value was set at 200 relative fluorescence units (RFU) above the noise band. The samples were tested in independent runs in separate rooms for a one-step real-time RT-PCR assay.

\section{Results}

\section{Phylogenetic analysis of PRRSV}

PRRSV was detected in 109 samples, which consisted of 47 samples for PRRSV-1 and 62 samples for PRRSV-2. Twenty samples were negative for PRRSV. Through ORF5 analysis, the PRRSV-1 samples belonged to pan-European subtype 1 and were classified into subgroup A (43/47, 91.49\%) and subgroup C (4/47, 8.51\%) (Fig. 1). The PRRSV-1 nucleotide sequence homologies of ORF5 to Lelystad were 79.74$87.23 \%$ and $90.69-94.78 \%$ for subgroup A and subgroup C, respectively. The nucleotide sequence 
homologies of ORF5, ORF6, and ORF7 among the different PRRSV-1 samples were 79.59-99.83\%, $85.85-100 \%$, and $86.52-100 \%$, respectively. The PRRSV-2 samples by ORF5 analysis were classified into lineage 1 (25/62, 40.32\%), Kor C (13/62, 20.97\%), Kor B (10/62, 16.13\%), lineage 5 (9/62, 14.52\%), and Kor A (5/62, 8.06\%) (Fig. 2A). The PRRSV-2 nucleotide sequence homologies of ORF5 to VR2332 were 79.88-82.52\%, 78.71-83.90\%, 84.63-85.77\%, 95.54-99.33\%, and $82.78-86.14 \%$ for lineage 1, Kor $C$ and $B$, lineage 5 , and Kor A, respectively. The nucleotide sequence homologies of ORF5, ORF6, and ORF7 among different samples of PRRSV-2 were $74.81-100 \%, 83.21-99.81 \%$, and $79.66-100 \%$, respectively. Among lineage 1, 22 samples (22/62, 35.48\%) could be classified into sublineage 1.8 (NADC30-like) and three samples $(3 / 62,4.84 \%)$ belonged to sublineage 1.6 (Fig. 2B). The mean evolutionary rates of 238 Korean PRRSV-1 and 319 PRRSV-2 were $6.931 \times 10^{-3} /$ site/year (95\% HPD intervals from $5.8521 \times 10^{-3}$ to $7.9748 \times 10^{-3}$ ) and $5.131 \times 10^{-3} /$ site/year ( $95 \%$ HPD intervals from $4.516 \times 10^{-3}$ to $\left.5.7664 \times 10^{-3}\right)$, respectively.

\section{Amino acid analysis of GP5 and M protein from PRRSV-1}

Previous studies identified one neutralizing epitope at amino acid (aa) 29-35 in GP5, which was reported to be ${ }^{29}$ WSFADGN ${ }^{35}$ in the Lelystad strain. Additionally, there were T cell epitopes and four B cell epitopes (GP5-I, GP5-II, GP5-III, and M-I) in GP5 and M protein of PRRSV-1 (48-51). As shown in Fig. 3, the neutralizing epitope was conserved with a low level of entropy. Subgroup A did not show much variation in the neutralizing epitope region, while only the 20R44-37-1 sample belonging to subgroup $C$ showed variation in position $31\left({ }^{31} \mathrm{~F} \rightarrow{ }^{31} \mathrm{~S}\right)$ and $35\left({ }^{35} \mathrm{~N} \rightarrow{ }^{35} \mathrm{~S}\right)$. By contrast, the GP5-III and M-I regions were variable with a high level of entropy, indicating genetic diversity (Fig. 3). A total of 16 codon sites were positively selected in the GP5 and M proteins of PRRSV-1 (Table 2). Interestingly, one positive selection site at position 35 was included in the neutralizing epitope region (positions 29-35), and two positive selection sites at positions 56 and 60 were included in the T cell epitope region (positions 53-75). Positive selection sites at positions $5,7,9,20,35,36$, and 104 in GP5 were observed with $p$-value $<0.01$.

\section{Amino acid analysis of GP5 and M protein from PRRSV-2}

Both a decoy epitope and a neutralizing epitope located at GP5 of PRRSV-2 have been identified, comprising residues $27-30\left({ }^{27} \mathrm{VLVN}^{30}\right)$ and residues $37-45\left({ }^{37} \mathrm{SHLQLIYNL}^{45}\right)$, respectively (Ostrowski et al., 2002). The diversity of amino acid sequences occurred in two previously identified B cell epitopes, aa 1-15 and aa 187-200, and two previously identified T cell epitopes, aa 117-131 and aa 149-163 (de Lima et al., 2006; Vashisht et al., 2008; Zhou et al., 2009). As shown in Fig. 4A, critical amino acid variations in the B cell and T cell epitopes were also found in GP5 of PRRSV-2. In the decoy epitope compared with the VR2332 strain, significant diversity was found with higher amino acid entropy. Interestingly, a specific substitution at position $44\left({ }^{44} \mathrm{~N} \rightarrow{ }^{44} \mathrm{~K}\right)$ was found in the 21R2-15-1 sample belonging to lineage 1 . In addition, 18R10-16-2 and 20D007-2 samples belonging to Kor $C$ and 21D82-1 sample belonging to lineage 1 had a cysteine at position 102 in GP5. The 18R10-16-2 sample belonging to Kor $C$ and 20D253-2 sample belonging to lineage 1 have an arginine at position 104 in GP5. Previous studies identified two B cell epitope regions at positions 10 and 70, and three T cell epitope regions at aa 
9-23, aa 33-47, and aa 57-71 of $\mathrm{M}$ proteins (52-54). The $\mathrm{M}$ protein region was relatively more conserved compared with that of GP5 (Fig. 4B). A specific substitution at position $8\left({ }^{8} \mathrm{~F} \rightarrow{ }^{8} \mathrm{~L}\right)$ was found in the 19R44-3-2 sample belonging to lineage 5. In addition, amino acid mutations and higher amino acid entropy at position 10 in 50 PRRSV-2 and position 70 in 11 PRRSV-2 were found. Positive selection pressure analysis confirmed 18 codon sites in GP5 (Table 2). Four positive selection sites at positions 2 , 5,6 , and 15 were included in the $B$ cell epitope region (positions 1-15), two positive selection sites at positions 28 and 30 were included in the decoy epitope region (positions 27-30), and five positive selection sites at positions 32, 33, 34, 35, and 59 were included in hypervariable regions 1 and 2. Subsequently, two positive selection sites at positions 102 and 104 were included in the B cell epitope regions, and one positive selection site at position 151 was included in the $T$ cell epitope region (positions 149-163). A total of four codon sites were found to be positively selected in M protein of PRRSV-2 (Table 2). One positive selection site at position 10 was included in the $B$ cell epitope region, and three positive selection sites at positions 15, 16, and 66 were included in the T cell epitope regions (Table 2).

\section{Sensitivities and specificities of three one-step real-time RT-PCR assays}

The sensitivities of three one-step real-time RT-PCR assays with two reference strains (Lelystad and LMY strain) were estimated to be $10^{0} \mathrm{TCID}_{50} / 100 \mu \mathrm{L}$. In the specificities of all one-step real-time RT-PCR assays determined by testing respiratory disease-causing viruses, such as PCV2, PCV3, CSFV, PPV, and SIV, no cross-reactivity was observed (data not shown).

\section{Clinical evaluation of three one-step real-time RT-PCR assays}

Twenty PRRSV-negative clinical samples showed the same results in all one-step real-time RT-PCR assays. As shown in Table 3, the A test detected $100 \%$ of PRRSV-1 (47/47) and PRRSV-2 (62/62) in the samples. The B test detected $100 \%$ of PRRSV-1 (47/47) and $98.39 \%$ of PRRSV-2 (61/62) in the samples. Only 18D283-1 sample, belonging to Kor A of PRRSV-2, was not detected in the B test. When identifying inhibitors in the 18D283-1 sample, none of the diluted samples was detected (data not shown). Meanwhile, the $C$ test detected $72.34 \%$ of PRRSV-1 (34/47) and $69.35 \%$ of PRRSV-2 (43/62) in the samples. There were 13 undetected samples of PRRSV-1 belonging to subgroups $\mathrm{A}(n=12)$ and subgroup $\mathrm{C}(n=1)$, and 19 undetected samples of PRRSV-2 belonged to $\operatorname{Kor} C(n=8), \operatorname{Kor} \mathrm{A}(n=4)$, lineage $1(n=4)$, Kor B $(n=2)$, and lineage $5(n=1)$. On comparison with the nucleotide sequences of the primers and probe in the $C$ test, several nucleotide mismatches were observed in the forward primer sequences. The forward primer against PRRSV-1 was located at positions 14,792-14,809 in ORF7 of the Lelystad strain. Several mismatches were observed of nucleotide positions 14,792 (G/A), 14,795 (C/T), 14,798 (C/T), 14,801 (C/T), 14,804 (C/T), 14,805 (C/T), 14,806 (A/T), and 14,808 (A/G). The forward primer against PRRSV-2 was located at positions 15,257-15,274 in 3' end of ORF7 and 3'-untranslated regions (UTR) of the VR2332 strain. Several mismatches were identified at nucleotide positions 15,277 (A/G), 15,261(T/C), 15,263(G/A/T), 15,265(C/T/A), 15,267(G/A), 15,268(G/T/A), 15,269(C/T), $15,270(\mathrm{~A} / \mathrm{G}) 15,271(\mathrm{~T} / \mathrm{C}), 15,272(\mathrm{~T} / \mathrm{C})$, and 15,274(C/T). In particular, the 18R10-52-1 sample, belonging to Kor $\mathrm{C}$ of PRRSV-2, has 15 nucleotide deletions in the detection region of the forward primer. 


\section{Discussion}

After 30 years of PRRSV emergence, PRRSV infection remains a critical disease that causes enormous economic losses to the swine industry worldwide. Despite widespread efforts to control and prevent PRRSV infection, the virus has rapidly spread worldwide and has increasing genetic diversity (55). The evolutionary rate of PRRSV (4.71-9.8 $\times 10^{-2} /$ sites/year) is the highest among RNA viruses (56), which allows genetic diversity within PRRSV and the emergence of new phenotypes. In this study, a phylogenetic analysis of Korean PRRSV was performed using clinical samples collected from January 2018 to September 2021. Our results are consistent with previous findings in which all Korean PRRSV-1 belong to pan-European subtype 1, and most belong to subgroup A. On the other hand, Korean PRRSV-2 belongs to lineage 1 , lineage 5 , and Korean lineages (Kor $A, B$, and $C$ ) and the majority of Korean PRRSV-2 belongs to lineage $5(13,41)$. However, in this study, lineage 1 shows the highest prevalence $(25 / 62$, $40.32 \%)$ followed by Kor C (13/62, 20.97\%), Kor B (10/62, 16.13\%), lineage $5(9 / 62,14.52 \%)$, and Kor A $(5 / 62,8.06 \%)$. A recent report showed that the PRRSV-2 lineage 1 population increased from 2014 (1.8\%) to 2019 (29.6\%) in Korea owing to the spread of sublineage 1.8 (NADC30-like viruses) and introduction of sublineage 1.6, comprising the second-largest population after lineage $5(31.1 \%)$ in $2019(41)$. These changes may support the hypothesis for these epidemic situations such as the importing of breeding pigs and artificial insemination $(57,58)$. Although the prevalence of PRRSV-2 in Korea should be confirmed in a larger sample size, we speculate that lineage 1 will become highly prevalent over time in Korea.

Previous studies showed ORF5 sequence homology of 85.8-90.9\% between Korean PRRSV-1 and Lelystad virus in 2005-2009, and 84.9-98.4\% in 2013-2016 (13). The ORF6 of Korean PRRSV-1 showed sequence homology of $93.2-98.6 \%$ among the Korean strains and $85.6-94.4 \%$ among the non-Korean strains in 2012 (59). The ORF7 of Korean PRRSV-1 showed a sequence homology of $88.8-99.7 \%$ among the Korean strains and $79.1-95.0 \%$ among the non-Korean strains in the $2000 \mathrm{~s}(11,14)$. In this study, the lowest nucleotide sequence homology of ORF5 between PRRSV-1 and the Lelystad strain was $79.74 \%$, indicating a decrease of $6.06 \%$ over approximately 15 years. The lowest homologies of ORF6 and ORF7 among the different PRRSV- 1 isolates were $85.85 \%$ and $86.52 \%$, respectively, indicating decreases of $7.35 \%$ and $2.28 \%$, respectively. The ORF5 sequence homology between Korean PRRSV-2 and VR2332 was $84.7-99.5 \%$ in $2003-2010$ and $82.3-99.3 \%$ in 2013-2016 (13). The ORF6 nucleotide sequence identity among the Korean PRRSV-2 strains was 85.5-98.2\% (60). The ORF7 of Korean PRRSV-2 showed a sequence homology of $86.2-100.0 \%$ with each other and $88.3-100 \%$ with isolates from other geographic regions (61). In this study, the lowest nucleotide sequence homology of ORF5 between PRRSV-2 and VR2332 was $78.71 \%$, indicating a decrease of $5.99 \%$ in over approximately 20 years. The lowest homologies of ORF6 and ORF7 among the different PRRSV-2 isolates is $83.21 \%$ and $79.66 \%$, respectively, indicating decreases of $2.29 \%$ and $6.54 \%$, respectively. Previous study showed nucleotide substitution rates of $1.46 \times 10^{-3}$ for PRRSV-2 viruses and $3.29 \times 10^{-3}$ substitutions/site/year for two genotype isolates based on ORF5 sequences data $(16,62)$ and $4.17-9.8 \times 10^{-2}$ substitutions/site/year based on ORFs 3-5 sequences of two genotype PRRSVs (56). Consistent with theses previous investigations, our 
results also indicate that Korean PRRSV has high substitution rates of $5.8521-7.9748 \times 10^{-3}$ for Korean PRRSV-1 and 4.516-5.7664 $\times 10^{-3}$ for Korean PRRSV-2. Therefore, it is suggested that the mutation rate of PRRSV circulating in Korea has increased over time.

In this study, the neutralizing epitope of PRRSV-1 was found to be conserved, but the GP5-III and M-I regions of PRRSV-1 were variable. Recent studies on the amino acid analysis of GP5 of Korean PRRSV-1 also showed a relatively conserved pattern in the B cell epitope regions, except for GP5-III (aa 165-176) $(13,41,63)$. GP5 and M of PRRSV-1 are not susceptible to antibody-mediated virus neutralization, in contrast to GP5 of PRRSV-2 is generally considered the main target for virus-neutralizing antibodies (50). To understand the mechanism of neutralizing antibody against the Korean PRRSV-1, further analysis of the neutralizing antibody-escape mutants of PRRSV in other minor envelope glycoproteins such as GP2, GP3, and GP4 is required. The residue at position 44 of PRRSV-2 GP5 plays a critical role in virus infectivity; position 50 of GP5 and position 8 of the M protein are essential for assembly of PRRSV particles $(64,65)$. In this study of PRRSV-2, the key residues at position 44 of GP5 and position 8 of M protein were variable in some samples. The key residues at positions 102 and 104 of PRRSV-2 GP5, which determine susceptibility to viral neutralization, were variable (66). Residues (V102C and G104R) that were identical to those in a neutralizing antibody-escape mutant and with higher amino acid entropy were found in our several samples under positive selective pressure. In addition, amino acid mutations at positions 10 and 70 of PRRSV-2 M protein are related to susceptibility to viral neutralization $(53,54)$; these features were validated in our samples. The hypervariable regions can modulate the accessibility of neutralizing antibodies to the neutralizing epitope (67). Recently, variability in the decoy epitope region and hypervariable regions of GP5, as well as mutations in key residues related to neutralizing antibodyescape mutants, have been commonly found in Korean PRRSV-2 amino acid analysis studies $(13,41$, $60)$. These variations were also found among the PRRSV-2 samples in this study. Therefore, Korean PRRSV-2 has evolved to genetic variants with resistance to neutralization and may be able to escape neutralization by antibodies that are induced by commercial PRRS modified live vaccines (MLV).

A positive selection signal was detected in the neutralizing epitope region of PRRSV-1. Incidentally, sequence analysis of PRRSV-2 revealed variation and positive selection pressure within the decoy epitope and the neutralizing epitope regions. Recent research demonstrated that vaccination resulted in the emergence of antibody-escaping mutants, in which strong positive selection contributed to amino acid substitutions (68). In a comparative analysis of PRRSV genetic diversity before and after vaccine adoption in Korea, PRRSV vaccination increased positively selected sites and the emergence of new glycosylation sites (63). Currently, several commercial PRRS MLVs are available in Korea for the control of PRRSV infections. In this respect, it can be inferred that PRRSV vaccination leads to affecting positive selection, resulting in the emergence of escape variants.

Several reports have shown that genetic variability of PRRSV resulting in mutations at the primer binding sites leads to failure of RT-PCR tests $(35,69)$. In the B test, only one sample (18D283-1) was not detected for PRRSV-2, which was not owing to an inhibitory effect. However, the nucleotide sequence could not be confirmed because the information on the primers and probe of the $B$ test was confidential. The $C$ test 
showed the lowest detection rate of $72.34 \%$ (34/47) for PRRSV-1 positive samples and $69.35 \%$ (43/62) for PRRSV-2 positive samples. On comparing the sequence of the false-negative samples of PRRSV-1 or PRRSV-2 and primers or probes of the $\mathrm{C}$ test, several nucleotide differences were found in several PRRSV1 and PRRSV-2 positive samples. In general, inconsistencies in diagnostic results are known to be more common in the detection of PRRSV-1 than PRRSV-2. This observation could be explained by the large genetic diversity of the viruses within the PRRSV-1 genotype $(35,44,70-72)$. However, this study showed similar proportions of inconsistencies in the detection of PRRSV-1 and PRRSV-2 samples in the C test, suggesting that the genetic diversity of the viruses within PRRSV-2 was also increased.

\section{Conclusions}

Our results demonstrate that two one-step real-time RT-PCR assays ( $A$ and $B$ tests) efficiently detected PRRSV-1 and PRRSV-2 in the clinical samples. However, considering the emergence of the dominant population, diversification of evolution within the epitope regions of PRRSV structural protein, and characteristics of PRRSV in which genetic mutations continue to occur, frequent evaluation of diagnostic methods is essential for an accurate diagnosis of PRRSV. In addition, regular monitoring of the emergence of new PRRSV will provide information on the implementation of control and preventive measures against PRRSV.

\section{Abbreviations}

PRRSV: porcine reproductive and respiratory syndrome virus; ORF: open reading frame; GP5: glycoprotein 5; M: membrane protein; RT-PCR: reverse transcription polymerase chain reactions; OIE: World Organization for Animal Health; Kor: Korean lineages; MAbs: monoclonal antibodies; SIV: swine influenza virus; APQA: Animal and Plant Quarantine Agency; $\operatorname{TCID}_{50} / \mathrm{ml}: 50 \%$ tissue culture infective dose per $\mathrm{ml}$; PCV2 and PCV3: porcine circovirus type 2 and type 3; CSFV: classical swine fever virus; PPV: porcine parvovirus; RFU: relative fluorescence units; aa: amino acid; MLV: modified live vaccines.

\section{Declarations}

\section{Ethics approval and consent to participate}

No ethical approval was required for the lung sample collection. Practicing veterinarians at local clinics collected tissue samples from the carcass during regular medical check-ups after receiving verbal consent from farm owners.

\section{Consent for publication}

Not applicable.

\section{Availability of data and materials}


The datasets supporting the conclusions of this article are included within the article.

\section{Competing interests}

The authors declare that they have no competing interests.

\section{Funding}

This work was supported by APQA (M-1543069-2021-22-01); the Korea Institute of Planning and Evaluation for Technology in Food, Agriculture and Forestry (IPET) through the Animal Disease Management Technology Development Program (321015-01-1-CG000); the Golden Seed Project (21301005-5-SB610 and PJ012818012021); and the Cooperative Research Program for Agriculture Science and Technology Development (Project No. PJ01561102) funded by the Ministry of Agriculture, Food and Rural Affairs (MAFRA), Rural Development Administration (RDA), Republic of Korea.

\section{Authors' contributions}

GES conducted the experiments and produced data and wrote the manuscript. JYP, MKK, and BKK contributed materials and data analysis. HYJ, KKL, and CKP participated in discussion and revises the manuscript. HYJ and CKP designed and supervised the study. All authors read and approved the final manuscript.

\section{Acknowledgements}

We would like to thank our funding sources.

\section{References}

1. Meulenberg JJ, Hulst MM, De Meijer EJ, Moonen PL, Den Besten A, De Kluyver EP, et al. Lelystad virus, the causative agent of porcine epidemic abortion and respiratory syndrome (PEARS), is related to LDV and EAV. Virology. 1993;192(1):62-72.

2. Neumann EJ, Kliebenstein JB, Johnson CD, Mabry JW, Bush EJ, Seitzinger AH, et al. Assessment of the economic impact of porcine reproductive and respiratory syndrome on swine production in the United States. Journal of the American Veterinary Medical Association. 2005;227(3):385-92.

3. Shi M, Lam TT-Y, Hon C-C, Hui RK-H, Faaberg KS, Wennblom T, et al. Molecular epidemiology of PRRSV: a phylogenetic perspective. Virus research. 2010;154(1-2):7-17.

4. Han M, Yoo D. Engineering the PRRS virus genome: updates and perspectives. Veterinary microbiology. 2014;174(3-4):279-95.

5. Wu W-H, Fang Y, Farwell R, Steffen-Bien M, Rowland RR, Christopher-Hennings J, et al. A 10-kDa structural protein of porcine reproductive and respiratory syndrome virus encoded by ORF2b. Virology. 2001;287(1):183-91. 
6. Johnson CR, Griggs TF, Gnanandarajah J, Murtaugh MP. Novel structural protein in porcine reproductive and respiratory syndrome virus encoded by an alternative ORF5 present in all arteriviruses. The Journal of general virology. 2011;92(Pt 5):1107.

7. Nelsen CJ, Murtaugh MP, Faaberg KS. Porcine reproductive and respiratory syndrome virus comparison: divergent evolution on two continents. Journal of virology. 1999;73(1):270-80.

8. Kweon C-h, Kwon B-j, Lee H-j, Cho J-j, Hwang E-k, Shin J-h, et al. Isolation of porcine reproductive and respiratory syndrome virus (PRRSV) in Korea. Korean Journal of Veterinary Research. 1994;34(1):7783.

9. Kim J-Y, Lee S-Y, Sur J-H, Lyoo YS. Serological and genetic characterization of the European strain of the porcine reproductive and respiratory syndrome virus isolated in Korea. Korean Journal of Veterinary Research. 2006;46(4):363-70.

10. Cha S-H, Choi E-J, Park J-H, Yoon S-R, Song J-Y, Kwon J-H, et al. Molecular characterization of recent Korean porcine reproductive and respiratory syndrome (PRRS) viruses and comparison to other Asian PRRS viruses. Veterinary microbiology. 2006;117(2-4):248-57.

11. Kim S-H, Roh I-S, Choi E-J, Lee C, Lee C-H, Lee K-H, et al. A molecular analysis of European porcine reproductive and respiratory syndrome virus isolated in South Korea. Veterinary microbiology. 2010;143(2-4):394-400.

12. Choi E-J, Lee C-H, Song J-Y, Song H-J, Park C-K, Kim B, et al. Genetic diversity of porcine reproductive and respiratory syndrome virus in Korea. Journal of veterinary science. 2013;14(2):115-24.

13. Kang H, Yu JE, Shin J-E, Kang A, Kim W-I, Lee C, et al. Geographic distribution and molecular analysis of porcine reproductive and respiratory syndrome viruses circulating in swine farms in the Republic of Korea between 2013 and 2016. BMC veterinary research. 2018;14(1):160.

14. Lee C, Kim H, Kang B, Yeom M, Han S, Moon H, et al. Prevalence and phylogenetic analysis of the isolated type I porcine reproductive and respiratory syndrome virus from 2007 to 2008 in Korea. Virus genes. 2010;40(2):225-30.

15. Thi Dieu Thuy N, Thi Thu N, Son NG, Ha LTT, Hung VK, Nguyen NT, et al. Genetic analysis of ORF 5 porcine reproductive and respiratory syndrome virus isolated in Vietnam. Microbiology and immunology. 2013;57(7):518-26.

16. Shi M, Lam TT-Y, Hon C-C, Murtaugh MP, Davies PR, Hui RK-H, et al. Phylogeny-based evolutionary, demographical, and geographical dissection of North American type 2 porcine reproductive and respiratory syndrome viruses. Journal of virology. 2010;84(17):8700-11.

17. Kang S-Y, Yun S-I, Park H-S, Park C-K, Choi H-S, Lee Y-M. Molecular characterization of PL97-1, the first Korean isolate of the porcine reproductive and respiratory syndrome virus. Virus research. 2004;104(2):165-79.

18. Egli C, Thür B, Liu L, Hofmann MA. Quantitative TaqMan® RT-PCR for the detection and differentiation of European and North American strains of porcine reproductive and respiratory syndrome virus. Journal of virological methods. 2001;98(1):63-75. 
19. Lurchachaiwong W, Payungporn S, Srisatidnarakul U, Mungkundar C, Theamboonlers A, Poovorawan $Y$. Rapid detection and strain identification of porcine reproductive and respiratory syndrome virus (PRRSV) by real-time RT-PCR. Letters in applied microbiology. 2008;46(1):55-60.

20. Balka G, Hornyák Á, Bálint Á, Benyeda Z, Rusvai M. Development of a one-step real-time quantitative PCR assay based on primer-probe energy transfer for the detection of porcine reproductive and respiratory syndrome virus. Journal of Virological Methods. 2009;158(1-2):41-5.

21. Rodríguez-Prieto V, Kukielka D, Martínez-López B, de las Heras Al, Barasona JÁ, Gortázar C, et al. Porcine reproductive and respiratory syndrome (PRRS) virus in wild boar and lberian pigs in southcentral Spain. European journal of wildlife research. 2013;59(6):859-67.

22. Stankevicius A, Buitkuviene J, Sutkiene V, Spancerniene U, Pampariene I, Pautienius A, et al. Detection and molecular characterization of porcine reproductive and respiratory syndrome virus in Lithuanian wild boar populations. Acta Veterinaria Scandinavica. 2015;58(1):51.

23. Dea S, Gagnon C, Mardassi H, Pirzadeh B, Rogan D. Current knowledge on the structural proteins of porcine reproductive and respiratory syndrome (PRRS) virus: comparison of the North American and European isolates. Archives of virology. 2000;145(4):659-88.

24. Gonin P, Pirzadeh B, Gagnon CA, Dea S. Seroneutralization of porcine reproductive and respiratory syndrome virus correlates with antibody response to the GP5 major envelope glycoprotein. Journal of Veterinary Diagnostic Investigation. 1999;11(1):20-6.

25. Meulenberg JJ, Petersen-Den Besten A, De Kluyver EP, Moormann RJ, Schaaper WM, Wensvoort G. Characterization of proteins encoded by ORFs 2 to 7 of Lelystad virus. Virology. 1995;206(1):155-63.

26. Mardassi H, Massie B, Dea S. Intracellular synthesis, processing, and transport of proteins encoded by ORFs 5 to 7 of porcine reproductive and respiratory syndrome virus. Virology. 1996;221(1):98-112.

27. Ostrowski M, Galeota J, Jar A, Platt K, Osorio FA, Lopez O. Identification of neutralizing and nonneutralizing epitopes in the porcine reproductive and respiratory syndrome virus GP5 ectodomain. Journal of virology. 2002;76(9):4241-50.

28. Fang L, Jiang Y, Xiao S, Niu C, Zhang H, Chen H. Enhanced immunogenicity of the modified GP5 of porcine reproductive and respiratory syndrome virus. Virus genes. 2006;32(1):5-11.

29. Wagstrom EA, Yoon K-J, Cook C, Zimmerman JJ. Diagnostic performance of a reverse transcriptionpolymerase chain reaction test for porcine reproductive and respiratory syndrome virus. Journal of veterinary diagnostic investigation. 2000;12(1):75-8.

30. Gerber PF, O'Neill K, Owolodun O, Wang C, Harmon K, Zhang J, et al. Comparison of commercial realtime reverse transcription-PCR assays for reliable, early, and rapid detection of heterologous strains of porcine reproductive and respiratory syndrome virus in experimentally infected or noninfected boars by use of different sample types. Journal of clinical microbiology. 2013;51(2):547-56.

31. OIE. Porcine Reproductive and Respipatory Syndrome: Manual of Diagnostic Tests and Vaccines for Terrestrial Animals. In World Organization for Animal Health (OIE).2015 [

32. Chang C-C, Yoon K-J, Zimmerman JJ, Harmon KM, Dixon PM, Dvorak C, et al. Evolution of porcine reproductive and respiratory syndrome virus during sequential passages in pigs. Journal of virology. 
2002;76(10):4750-63.

33. Klungthong C, Chinnawirotpisan P, Hussem K, Phonpakobsin T, Manasatienkij W, Ajariyakhajorn C, et al. The impact of primer and probe-template mismatches on the sensitivity of pandemic influenza A/H1N1/2009 virus detection by real-time RT-PCR. Journal of Clinical Virology. 2010;48(2):91-5.

34. Toplak I, Rihtarič D, Hostnik P, Grom J, Štukelj M, Valenčak Z. Identification of a genetically diverse sequence of porcine reproductive and respiratory syndrome virus in Slovenia and the impact on the sensitivity of four molecular tests. Journal of virological methods. 2012;179(1):51-6.

35. Wernike K, Bonilauri P, Dauber M, Errington J, LeBlanc N, Revilla-Fernández S, et al. Porcine reproductive and respiratory syndrome virus: interlaboratory ring trial to evaluate real-time reverse transcription polymerase chain reaction detection methods. Journal of veterinary diagnostic investigation. 2012;24(5):855-66.

36. Christopher-Hennings J, Faaberg KS, Murtaugh MP, Nelson EA, Roof MB, Vaughn EM, et al. Porcine reproductive and respiratory syndrome (PRRS) diagnostics: Interpretation and limitations. Journal of Swine Health and Production. 2002;10(5):213-8.

37. Papin JF, Vahrson W, Dittmer DP. SYBR green-based real-time quantitative PCR assay for detection of West Nile Virus circumvents false-negative results due to strain variability. Journal of Clinical Microbiology. 2004;42(4):1511-8.

38. Fetzer C, Pesch S, Ohlinger V. High risk of false positive results in a widely used diagnostic test for detection of the porcine reproductive and respiratory syndrome virus (PRRSV). Veterinary microbiology. 2006;115(1-3):21-31.

39. Kuo H, Lo D, Chen C, Lee C, Shen Y, Tsai Y, et al. Rapid detection of type 2 porcine reproductive and respiratory syndrome virus by a duplex reverse transcription insulated isothermal PCR on a fielddeployable system. J Vet Sci Technol. 2017;8(415):2.

40. Alkhamis MA, Perez AM, Murtaugh MP, Wang X, Morrison RB. Applications of Bayesian phylodynamic methods in a recent US porcine reproductive and respiratory syndrome virus outbreak. Frontiers in microbiology. 2016;7:67.

41. Kim S-C, Jeong C-G, Park G-S, Park J-Y, Jeoung H-Y, Shin G-E, et al. Temporal lineage dynamics of the ORF5 gene of porcine reproductive and respiratory syndrome virus in Korea in 2014-2019. Archives of Virology. 2021:1-13.

42. Pond SLK, Frost SD. Datamonkey: rapid detection of selective pressure on individual sites of codon alignments. Bioinformatics. 2005;21(10):2531-3.

43. Murrell B, Wertheim JO, Moola S, Weighill T, Scheffler K, Kosakovsky Pond SL. Detecting individual sites subject to episodic diversifying selection. PLoS genetics. 2012;8(7):e1002764.

44. OIE. Porcine Reproductive and Respipatory Syndrome: Manual of Diagnostic Tests and Vaccines for Terrestrial Animals. 2015 [updated May 2015.

45. Kleiboeker SB, Schommer SK, Lee S-M, Watkins S, Chittick W, Polson D. Simultaneous detection of North American and European porcine reproductive and respiratory syndrome virus using real-time 
quantitative reverse transcriptase-PCR. Journal of Veterinary Diagnostic Investigation. 2005;17(2):165-70.

46. Wilson IG. Inhibition and facilitation of nucleic acid amplification. Applied and environmental microbiology. 1997;63(10):3741-51.

47. Gutiérrez-Aguirre I, Steyer A, Boben J, Gruden K, Poljsak-Prijatelj M, Ravnikar M. Sensitive detection of multiple rotavirus genotypes with a single reverse transcription-real-time quantitative PCR assay. Journal of clinical microbiology. 2008;46(8):2547-54.

48. Wissink E, van Wijk H, Kroese M, Weiland E, Meulenberg J, Rottier P, et al. The major envelope protein, GP5, of a European porcine reproductive and respiratory syndrome virus contains a neutralization epitope in its N-terminal ectodomain. Journal of General Virology. 2003;84(6):1535-43.

49. Díaz I, Pujols J, Ganges L, Gimeno M, Darwich L, Domingo M, et al. In silico prediction and ex vivo evaluation of potential T-cell epitopes in glycoproteins 4 and 5 and nucleocapsid protein of genotypeI (European) of porcine reproductive and respiratory syndrome virus. Vaccine. 2009;27(41):5603-11.

50. Vanhee M, Van Breedam W, Costers S, Geldhof M, Noppe Y, Nauwynck H. Characterization of antigenic regions in the porcine reproductive and respiratory syndrome virus by the use of peptidespecific serum antibodies. Vaccine. 2011;29(29-30):4794-804.

51. Mokhtar H, Eck M, Morgan SB, Essler SE, Frossard J-P, Ruggli N, et al. Proteome-wide screening of the European porcine reproductive and respiratory syndrome virus reveals a broad range of $\mathrm{T}$ cell antigen reactivity. Vaccine. 2014;32(50):6828-37.

52. Wang Y-x, Zhou Y-j, Li G-x, Zhang S-r, Jiang Y-f, Xu A-t, et al. Identification of immunodominant T-cell epitopes in membrane protein of highly pathogenic porcine reproductive and respiratory syndrome virus. Virus research. 2011;158(1-2):108-15.

53. Trible BR, Popescu LN, Monday N, Calvert JG, Rowland RR. A single amino acid deletion in the matrix protein of porcine reproductive and respiratory syndrome virus confers resistance to a polyclonal swine antibody with broadly neutralizing activity. Journal of virology. 2015;89(12):6515-20.

54. Fan B, Liu X, Bai J, Zhang T, Zhang Q, Jiang P. Influence of the amino acid residues at 70 in $M$ protein of porcine reproductive and respiratory syndrome virus on viral neutralization susceptibility to the serum antibody. Virology journal. 2016;13(1):1-11.

55. Murtaugh MP, Stadejek T, Abrahante JE, Lam TT, Leung FC-C. The ever-expanding diversity of porcine reproductive and respiratory syndrome virus. Virus research. 2010;154(1-2):18-30.

56. Hanada K, Suzuki Y, Nakane T, Hirose O, Gojobori T. The origin and evolution of porcine reproductive and respiratory syndrome viruses. Molecular biology and evolution. 2005;22(4):1024-31.

57. Zhou L, Wang Z, Ding Y, Ge X, Guo X, Yang H. NADC30-like strain of porcine reproductive and respiratory syndrome virus, China. Emerging infectious diseases. 2015;21(12):2256.

58. Sun YK, Chen YJ, Cai Y, Li Q, Xie JX, Liang G, et al. Insights into the evolutionary history and epidemiological characteristics of the emerging lineage 1 porcine reproductive and respiratory syndrome viruses in China. Transboundary and emerging diseases. 2020;67(6):2630-41. 
59. Lee D-U, Yoo SJ, Kwon T, Sang HJ, Shin JY, Byun JJ, et al. Genetic diversity of ORF 4-6 of type 1 porcine reproductive and respiratory syndrome virus in naturally infected pigs. Veterinary microbiology. 2017;199:54-61.

60. Kwon T, Yoo SJ, Sunwoo SY, Lee D-U, Sang HJ, Park JW, et al. Independent evolution of porcine reproductive and respiratory syndrome virus 2 with genetic heterogeneity in antigenic regions of structural proteins in Korea. Archives of virology. 2019;164(1):213-24.

61. Yoon S, Song J-Y, Lee C-H, Choi E-J, Cho I, Kim B. Genetic characterization of the Korean porcine reproductive and respiratory syndrome viruses based on the nucleocapsid protein gene (ORF7) sequences. Archives of virology. 2008;153(4):627-35.

62. Song J, Shen D, Cui J, Zhao B. Accelerated evolution of PRRSV during recent outbreaks in China. Virus genes. 2010;41(2):241-5.

63. Kwon T, Yoo SJ, Lee D-U, Sunwoo SY, Sang HJ, Park JW, et al. Differential evolution of antigenic regions of porcine reproductive and respiratory syndrome virus 1 before and after vaccine introduction. Virus research. 2019;260:12-9.

64. Verheije M, Welting T, Jansen $H$, Rottier P, Meulenberg J. Chimeric arteriviruses generated by swapping of the $\mathrm{M}$ protein ectodomain rule out a role of this domain in viral targeting. Virology. 2002;303(2):364-73.

65. Ansari IH, Kwon B, Osorio FA, Pattnaik AK. Influence of N-linked glycosylation of porcine reproductive and respiratory syndrome virus GP5 on virus infectivity, antigenicity, and ability to induce neutralizing antibodies. Journal of virology. 2006;80(8):3994-4004.

66. Fan B, Liu X, Bai J, Zhang T, Zhang Q, Jiang P. The amino acid residues at 102 and 104 in GP5 of porcine reproductive and respiratory syndrome virus regulate viral neutralization susceptibility to the porcine serum neutralizing antibody. Virus research. 2015;204:21-30.

67. Popescu LN, Trible BR, Chen N, Rowland RR. GP5 of porcine reproductive and respiratory syndrome virus (PRRSV) as a target for homologous and broadly neutralizing antibodies. Veterinary microbiology. 2017;209:90-6.

68. Costers S, Vanhee M, Van Breedam W, Van Doorsselaere J, Geldhof M, Nauwynck HJ. GP4-specific neutralizing antibodies might be a driving force in PRRSV evolution. Virus research. 2010;154(12):104-13.

69. Harmon KM, Abate SA, Chriswell AJ, Chittick WA, Strait EL. Comparison of two commercial real-time reverse transcriptase polymerase chain reaction assays for porcine reproductive and respiratory syndrome virus. Journal of Swine Health and Production. 2012;20(4):184-8.

70. Stadejek T, Stankevicius A, Storgaard T, Oleksiewicz MB, Belak S, Drew T, et al. Identification of radically different variants of porcine reproductive and respiratory syndrome virus in Eastern Europe: towards a common ancestor for European and American viruses. Journal of General Virology. 2002;83(8):1861-73.

71. Pesch S, Meyer C, Ohlinger V. New insights into the genetic diversity of European porcine reproductive and respiratory syndrome virus (PRRSV). Veterinary microbiology. 2005;107(1-2):31-48. 
72. Darwich L, Gimeno M, Sibila M, Diaz I, de la Torre E, Dotti S, et al. Genetic and immunobiological diversities of porcine reproductive and respiratory syndrome genotype I strains. Veterinary microbiology. 2011;150(1-2):49-62.

\section{Tables}

Table 1. Primers for RT-PCR amplification of ORF 5, ORF6, and ORF7 genes of PRRSV

\begin{tabular}{|c|c|c|c|c|}
\hline Genotype & Target & Primer & Sequence $5^{\prime}-3^{\prime}$ & Reference \\
\hline \multirow[t]{6}{*}{ PRRSV-1 } & \multirow[t]{2}{*}{ ORF5 } & EU ORF5 F & CCGTCTGTGATGAGRTGGGC & \multirow[t]{2}{*}{ Kang et al., 2018} \\
\hline & & EU ORF5 R & GGAYACTTTTAGGGCRTATA & \\
\hline & \multirow[t]{2}{*}{ ORF6 } & EU ORF6 F & GTCGTCCTCGAAGGGGTTAAAG & \multirow[t]{10}{*}{ In this study ${ }^{a}$} \\
\hline & & EU ORF6 R & YGGCGCTGGGACTTYATCA & \\
\hline & \multirow[t]{2}{*}{ ORF7 } & EU ORF7 F & GCATACGCTGTGAGAAAGC & \\
\hline & & EU ORF7 R & CTATTCAATTAGGGCGACCGTG & \\
\hline \multirow[t]{6}{*}{ PRRSV-2 } & \multirow[t]{2}{*}{ ORF5 } & NA ORF5 $\mathrm{F}$ & GTGGGCRACYGTTTTAGCCT & \\
\hline & & NA ORF5 R & CATAGTGAGCGCGACCYTAT & \\
\hline & \multirow[t]{2}{*}{ ORF6 } & NA ORF6 $\mathrm{F}$ & TYGTGCTTGATGGTTCCGYG & \\
\hline & & NA ORF6 R & AGYTGATTGACTGGCTGGCC & \\
\hline & \multirow[t]{2}{*}{ ORF7 } & NA ORF7 F & AACGGYACAYTGGTGCCC & \\
\hline & & NA ORF7 R & CTATTCAATTAGGGCGACCGTG & \\
\hline
\end{tabular}

a The target regions of Korean PRRSV-1 and PRRSV-2 strains collected from the NCBI database were aligned and the primer were designed in conserved sequences of target genes.

Table 2. Positive selection sites of Korean PRRSV identified between January 2018 and September 2021 


\begin{tabular}{|c|c|c|c|c|c|c|c|c|}
\hline Type & PRRS & & & & PRRS & & & \\
\hline Structural protein & GP5 & & $M$ & & GP5 & & $M$ & \\
\hline Positively selected sites & Site ${ }^{a}$ & P-value & Site $^{a}$ & P-value & Site ${ }^{b}$ & P-value & Site $^{b}$ & P-value \\
\hline & 2 & 0.03 & 58 & 0.02 & 2 & 0.03 & 10 & $<0.01$ \\
\hline & 5 & $<0.01$ & & & 5 & 0.02 & 15 & 0.03 \\
\hline & 7 & $<0.01$ & & & 6 & 0.03 & 16 & $<0.01$ \\
\hline & 8 & 0.02 & & & 15 & 0.04 & 66 & $<0.01$ \\
\hline & 9 & $<0.01$ & & & 26 & 0.03 & & \\
\hline & 12 & 0.04 & & & 28 & 0.01 & & \\
\hline & 14 & 0.03 & & & 30 & 0.04 & & \\
\hline & 20 & $<0.01$ & & & 32 & $<0.01$ & & \\
\hline & 22 & 0.04 & & & 33 & 0.01 & & \\
\hline & 35 & $<0.01$ & & & 34 & $<0.01$ & & \\
\hline & 36 & $<0.01$ & & & 35 & 0.02 & & \\
\hline & 56 & 0.02 & & & 59 & 0.04 & & \\
\hline & 60 & 0.01 & & & 61 & 0.03 & & \\
\hline & 103 & 0.03 & & & 73 & 0.02 & & \\
\hline & 104 & $<0.01$ & & & 98 & 0.02 & & \\
\hline & & & & & 102 & 0.05 & & \\
\hline & & & & & 104 & 0.05 & & \\
\hline & & & & & 151 & 0.05 & & \\
\hline
\end{tabular}

${ }^{a}$ Amino acid position based on Lelystad strain.

${ }^{\mathrm{b}}$ Amino acid position based on VR2332 strain

Table 3. Comparison of three one-step real-time RT-PCR with 47 PRRSV-1 and 62 PRRSV-2 
Genotype Number of samples Number of positive (\%)

\begin{tabular}{lllll} 
& & A test & B test & C test \\
\hline PRRSV-1 & 47 & $47(100)$ & $47(100)$ & $34(72.34)$ \\
\hline PRRSV-2 & 62 & $62(100)$ & $61(98.39)$ & $43(69.35)$ \\
\hline Total & 109 & $109(100)$ & $108(99.08)$ & $77(70.64)$
\end{tabular}

\section{Figures}

\section{Figure 1}

Phylogenetic analysis of ORF5 of PRRSV-1 isolates. The tree was constructed by maximum likelihood

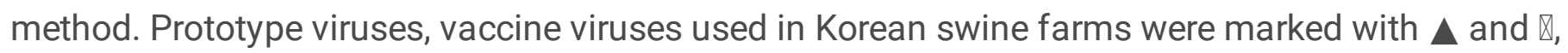
respectively. Subgroup $A$ and $C$ of field strains obtained from this study was marked with $\boldsymbol{\Delta}$ and $\boldsymbol{O}$, respectively.

\section{Figure 2}

Phylogenetic analysis of ORF5 of PRRSV-2 isolates (A). The tree was constructed by maximum likelihood

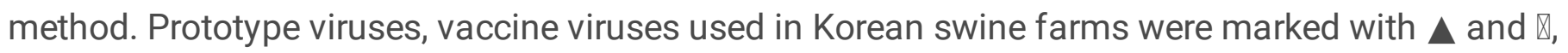
respectively. Lineage 1 , lineage 5 , Kor $A$, Kor $B$, and Kor $C$ of field strains obtained from this study was

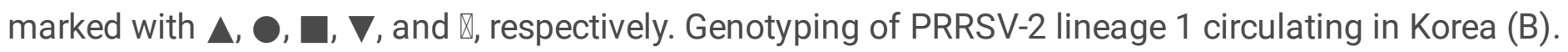

\section{Figure 3}

The alignment and entropy plot (amino acid diversity) of GP5 and M of PRRSV-1 samples with Lelystad strain. Multiple alignments of GP5 (A) and M (B) protein of PRRSV-1 are numbered from start of the GP5 domain (aa 1-201) and $M$ protein domain (aa 1-100), respectively. The sequence of Lelystad is shown at the top of $(A)$ and $(B)$. The size of each aa letter at each sequence logos is proportional to the frequency. Amino acids are color-coded: blank, nonpolar; green, polar uncharged; red, polar with a positive charge; blue, polar with negative charge. A red square box indicates a B-cell epitope region, and a red round box indicates a T-cell epitope region. NE: neutralizing epitope, T epitope: T-cell epitope. 
The alignment and entropy plot (amino acid diversity) of GP5 and M of PRRSV-2 samples with VR2332 strain. Multiple alignments of GP5 (A) and M (B) protein of PRRSV-2 are numbered from start of the GP5 domain (aa 1-200) and M protein domain (aa 1-100), respectively. The sequence of VR2332 is shown at the top of $(A)$ and $(B)$. The meaning of the size and color of the sequence logos is explained in the legend to Fig.3. A red square box indicates a B-cell epitope region, and a red round box indicates a T-cell epitope region. DE: decoy epitope, NE: neutralizing epitope, HVR: hypervariable region, $B$ epitope: B-cell epitope, $T$ epitope: T-cell epitope. 